\title{
Neoadjuvant chemotherapy alters the balance of effector to suppressor immune cells in advanced ovarian cancer
}

\author{
Alexandra Leary ${ }^{1,6}\left(\right.$ Catherine Genestie $^{2} \cdot$ Félix Blanc-Durand $^{1} \cdot$ Sébastien Gouy ${ }^{1}$. Ariane Dunant ${ }^{3}$. \\ Amandine Maulard ${ }^{1}$. Françoise Drusch ${ }^{2}$. Bianca Cheaib ${ }^{5}$. Judith Michels ${ }^{1}$ - Enrica Bentivegna ${ }^{1}$ - Audrey LeFormal ${ }^{4}$. \\ Soizick Mesnage ${ }^{4} \cdot$ Philippe Morice $^{1} \cdot$ Patricia Pautier $^{1} \cdot$ Aya S. Khairallah $^{2}$
}

Received: 6 February 2020 / Accepted: 9 July 2020 / Published online: 27 August 2020

(c) The Author(s) 2020

\begin{abstract}
Background At diagnosis, tumor-infiltrating lymphocytes (TILs) are prognostic in epithelial ovarian cancer (EOC). We recently demonstrated that neoadjuvant chemotherapy (NACT) significantly increased stromal TILs. Here, we investigated the impact of NACT on immune subpopulations with a particular focus on the balance of immune-reactive to tolerant subpopulations.

Materials and methods Tissue microarrays of EOC (145 pre-NACT, 139 post-NACT) were analyzed for CD3+, CD8+, FOXP3+, CD68+, and CD163+ by immunohistochemistry and CD4+ cells from deduction. Stromal TILs scored as percentage of stromal area, while intra-epithelial TILs scored as number of TILs in contact with tumor cells/HPF. Differences were evaluated by Wilcoxon or Chi square tests, Wilcoxon signed-rank for paired analyses, and cox model for PFS and OS. Results NACT significantly increased stromal CD3+ $(p=0.003)$ and CD8+ $(p=0.001)$ and intra-epithelial CD8+ $(p=0.022)$ and CD68+ $(p=0.0003)$ infiltration in unmatched samples and among paired samples for stromal CD3+ and CD8+. Neither $\mathrm{CD} 3+, \mathrm{CD} 8+, \mathrm{CD} 4+$, and CD68+ nor CD163+ expression correlated with outcome at diagnosis or post NACT. Using median value as a cut-off, high stromal CD8+/FOXP3+ ratio $(\mathrm{HR}=0.59 ; p=0.017)$ and high stromal $\mathrm{CD} 3+/ \mathrm{FOXP} 3+$ ratio post NACT were associated with prolonged PFS $(p=0.0226)$. The more the balance shifted in favor of effector versus regulatory TILs, the better the survival. Similarly, high CD68+/CD163+ ratio post NACT improved PFS $(p=0.0445)$.

Conclusion NACT has a significant impact on the balance of immune-reactive to immune-tolerant subpopulations and a high ratio of $\mathrm{CD} 8+/ \mathrm{FOXP} 3+, \mathrm{CD} 3+/ \mathrm{FOXP} 3+$, and $\mathrm{CD} 68+/ \mathrm{CD} 163+$ post $\mathrm{NACT}$ was significantly associated with improved outcomes. Whether this could select patients for immunotherapy in the post-operative setting should be investigated.
\end{abstract}

Keywords Ovarian cancer $\cdot$ Microenvironment $\cdot$ TILs $\cdot$ Neoadjuvant chemotherapy $\cdot$ Immunotherapy

Electronic supplementary material The online version of this article (https://doi.org/10.1007/s00262-020-02670-0) contains supplementary material, which is available to authorized users.

Alexandra Leary

Alexandra.leary@gustaveroussy.fr

1 Gynecological Cancer Unit, Department of Medicine, Institut Gustave Roussy, Villejuif, France

2 Pathology Department, Institut Gustave Roussy, Villejuif, France

3 Biostatistics and Epidemiology Unit, Institut Gustave Roussy, Villejuif, France

\section{Background}

The majority of patients with epithelial ovarian cancer (EOC) present with advanced tumor stage (FIGOIII/IV) propelling it to the most lethal gynecologic malignancy. Indeed, EOC affects approximately 1 in 70 women with only

4 INSERM U981, Institut Gustave Roussy, Villejuif, France

5 Department of Cancer Medicine, Breast Cancer Committee, Institut Gustave Roussy, Villejuif, France

6 Medical Oncologist, Gynecology Unit, INSERM U981, Institut Gustave Roussy, 114 rue Edouard Vaillant, 94805 Villejuif, France 
$45 \%$ surviving 5 years after diagnosis [1]. The backbone of management rests on complete resection of all macroscopic disease and platinum-based chemotherapy [2]. In advanced disease where complete resection cannot be achieved, neoadjuvant chemotherapy (NACT) followed by interval debulking surgery and adjuvant chemotherapy is a suitable alternative $[3,4]$.

There is increasing evidence suggesting that measures of local anti-tumor immunity may be relevant prognostic factors in several neoplasms including ovarian cancer. Tumor-infiltrating lymphocytes (TILs) have been repeatedly associated with improved survival in EOC [5-8]. Infiltration of CD3+ T-cells, and particularly CD8+ cytotoxic cells, is most strongly and consistently associated with a better prognosis in many independent studies [5, 7, 9-14].

On the other hand, immune-suppressive cells, such as FOXP3+ regulatory T-cells (Tregs), also present in the tumor bed, are often associated with a negative prognostic impact [5, 15]. Similarly, tumor-associated macrophages (TAMs) have been shown to predict an unfavorable prognosis, in particular, the M2-polarized (CD163+) subset [5, 16]. In fact, the interaction between the immune system and the tumor is likely based on an equilibrium between immune recognition and immune tolerance so that a measure of the balance between immune activating and immune-suppressive cells may be more informative. Accordingly, studies have reported that high ratios of intra-tumoral CD8/Tregs or CD68/CD163 macrophages were associated with improved overall survival in ovarian cancer $[5,16,17]$.

Unfortunately, most studies have focused on chemotherapy naïve tumors. Emerging data suggest that conventional cytotoxics may alter the local immune state by inducing adaptive stress response in malignant cells, hence operating as immunological adjuvant, or by depleting tumor-infiltrating immunosuppressive cells [18-20].

The neoadjuvant setting where paired ovarian tumor samples are obtained at diagnosis and at interval debulking after platinum-based chemotherapy offers a useful model to study the impact of cytotoxic treatment on anti-tumor immunity. We have recently shown that neoadjuvant chemotherapy (NACT) had a significant impact on the ovarian tumor immune microenvironment by increasing TIL infiltration and PDL1 expression [21]. It is, therefore, crucial to define which immune subsets are actually recruited to the tumor bed post NACT, and specifically whether these are involved in immune surveillance or immune tolerance.

Our aim was to further elucidate the impact of chemotherapy on the tumor immune landscape by characterizing the changes in subsets of T-cells and macrophages with NACT in a large cohort of clinically annotated ovarian cancer samples before and after NACT with a particular focus on the balance of putative immune-reactive to immune-tolerant subpopulations. Immune parameters before and after NACT were also related to clinical outcome to evaluate their prognostic value.

\section{Materials and methods}

\section{Tissue microarray (TMA)}

A clinically annotated TMA of epithelial ovarian cancer (EOC) samples from the Gustave Roussy Cancer Center biobank was constructed as previously published from 150 patients providing 133 pre-chemotherapy samples obtained at diagnostic laparoscopy or primary surgery and 128 postchemotherapy samples obtained at interval surgery. Samples were selected based on the greatest viable tumor cellularity; necrotic samples were excluded. In few patients with complete pathological response, immune parameters were not evaluated. The presence of tumor in the arrayed samples was confirmed by H\&E staining.

\section{Immunohistochemistry}

Immunohistochemical staining of TMA slides of formalinor acetic acid formalin alcohol (AFA)-fixed samples was performed by the autostainer Benchmark Ultra (ROCHE). The slides were dewaxed and rehydrated. Antigen retrieval was performed using a citrate-based antigen unmasking solution (CC1) for $36 \mathrm{~min}$. The following antibodies were used with UltraView Kit: rabbit anti-human CD3 antibody (DAKO; clone:polyclonal) (1/100) for $1 \mathrm{~h}$ at $37{ }^{\circ} \mathrm{C}$, rabbit anti-human CD8 antibody (Spring Bioscience; clone: SP16) (1/100) for $1 \mathrm{~h}$ at $37^{\circ} \mathrm{C}$, rabbit anti-human FOXP3 antibody (Spring Bioscience; clone: SP97) (1/100) for $1 \mathrm{~h}$ at $37^{\circ} \mathrm{C}$ with ampli, mouse anti-human CD68 antibody (DAKO; clone:KP1) (1/1000) for $20 \mathrm{~min}$ at $37{ }^{\circ} \mathrm{C}$, and mouse antihuman CD163 antibody (DPS, Cliniscience; clone:10D6) (1/75) for $44 \mathrm{~min}$ at room temperature.

\section{Histopathological analysis and TILs assessment}

Immunostained TMAs were examined and scored by two pathologists (A.K. and C.G.) blinded to clinical data. For every marker, a mean score from three cores from each sample was given after evaluation at $20 \times$ and $40 \times$ objective lens. The assessment of marked TILs was done following the guidelines described by the international TILs Working Group 2014 [22] : The boundaries of the invasive tumor were identified and only TILs inside them were evaluated (Supplementary Fig. 1a). Stromal TILs (sTILs) were defined as lymphocytes located in the tumor stroma between the carcinoma cells, but with no direct contact with them, while intra-epithelial TILs (ieTILs) were defined as lymphocytes 
in tumor nests having cell-to-cell contact with no intervening stroma and in direct interaction with carcinoma cells (Supplementary Fig. 1b). Since both types of TILs are localized in the region defined as tumor tissue, both categories represent true TILs.

Stromal subsets of TILs were scored as the percentage of total intra-tumoral stromal area occupied by CD3+, CD8+, or FOXP3+ mononuclear cells. Since no international recommendations are available for intra-epithelial TILs (ieTILs) scoring and methodologies vary widely in previous reports, they were quantified by counting the average of number of intra-epithelial TILs per high-power field $(\times 40)$ on 5 fields. CD4+ stromal and ieTILs were estimated by subtracting $\mathrm{CD} 8+$ cells from $\mathrm{CD} 3+$ cells.

Since TMA spots were lost with each additional section, both macrophage markers (CD68 in red and CD163 in brown) were performed on the same slide by double labeling and were determined as the percentage of positive cells within the tumor area. Because of the doublestaining, the boundaries between stroma, macrophages, and epithelial cells were often unclear, so CD68 and CD163 scoring encompassed both stromal and intra-epithelial compartments.

TILs in areas with crush artifacts, necrosis, or extensive regressive hyalinization were not scored. A necrotic biopsy was considered not assessable.

\section{Statistical analysis}

All statistical analyses were performed using SAS 9.3. The cut-off to define high/low for each marker was the median value rounded to the nearest integer. Continuous variables were compared with Wilcoxon tests. Comparisons of categorical variables were performed using Chi square tests which were exact when appropriate. For paired samples, the Wilcoxon rank sign test was used, after checking that for most differences of marker expressions, the normality assumption was violated.

Progression-free survival (PFS) was calculated from the date of histological diagnosis to progression or death. Survival curves were generated using the Kaplan-Meier Method and analyses were performed with a Cox model.

\section{Results}

\section{Clinical patient profile}

The clinical and histological characteristics of patients included in this study were previously published (Supplementary Table 1) [21]. In brief, patients with newly diagnosed Stage III/IV epithelial ovarian cancer were treated with platinum and paclitaxel neoadjuvant chemotherapy (mean number of cycles $=4$ ) with the goal of achieving complete cytoreduction. The median duration of follow-up was 80 months (95\% CI 68-93 months), median PFS for the whole cohort was 20 months (95\% CI 18-23 months), and median overall survival (OS) was 45 months $(95 \% \mathrm{CI}$ 39-62 months).

\section{Changes in T-cell subsets with NACT}

The number of samples evaluable for each marker varied due to crush artifacts, or because samples were lost as the TMA was increasingly sectioned.

Total CD3+ T-cells, as well as cytotoxic CD8+, helper $\mathrm{CD} 4+$, and regulatory FOXP3+ T-cells were evaluated in the stromal and intra-epithelial compartments in tumor samples before and after NACT.

At diagnosis, stromal CD3+ TILs were detected in all tumor samples but showed wide variability among tumor samples $(N=110$, median: $15 \%$; range $1-57 \%)$. This interpatient heterogeneity was also observed for stromal CD8+ $(N=107$, median: $10 \%$; range $0-40 \%), \mathrm{CD} 4+(N=106$, median: 5\%; range $0-32 \%)$, and FOXP3+ TILs $(N=86$, median: 5\%; range 0-25\%). In contrast, intra-epithelial TILs were found in fewer cases. Median for intra-epithelial CD3+ (range 0-30 cells/HPF) was 2 cells/HPF, and only 1 cell/ HPF for CD8+ (range 0-18 cells/HPF), CD4+ (range 0-19 cells/HPF), and FOXP3+ TILs (range 0-7 cells/HPF).

Overall, stromal CD3+ T-cells increased significantly after NACT (median: $15 \%$ to $20 \%$ pre to post; $p=0.003$, Fig. 1a). Similar increases were noted in stromal CD8+ TILs ( $p=0.001$, Fig. $1 \mathrm{~b})$ and in CD8+ ieTILs $(p=0.022$, Fig. 1c). There was no significant difference in median CD4 or FOXP3 stromal/intra-epithelial scores before and after NACT (data not shown).

Among the smaller subset of patients with matched paired pre- and post-NACT samples, a significant increase in median stromal CD3+ $(p=0.02, N=77)$ and stromal $\mathrm{CD} 8+(p=0.008, N=73)$ was also observed. However, the impact of NACT was clearly variable among individual patients with 55\% of the patients (43/77) showing increased, $37 \%$ (29/77) showing decreased, and 8\% (6/77) showing unchanged stromal CD3+ TILs post NACT (Fig. 2a-e). Similar patterns were observed with stromal CD8+ TILs (Fig. 3a-f) highlighting the inter-tumor variability in immune responses to NACT.

With regards to FOXP3+ TIL infiltration, there was no overall difference in the median stromal or intra-epithelial scores; however, when considering paired samples from individual patients, the pattern of change in FOXP3+ infiltration varied markedly among patients. Half the patients (27/54) showed decreasing FOXP3+ stromal infiltration, while 38\% demonstrated stromal FOXP3 + + TIL recruitment post NACT. With regards to ieFOXP3+ TILs, 33\% exhibited increasing infiltration, while $50 \%$ of patients 


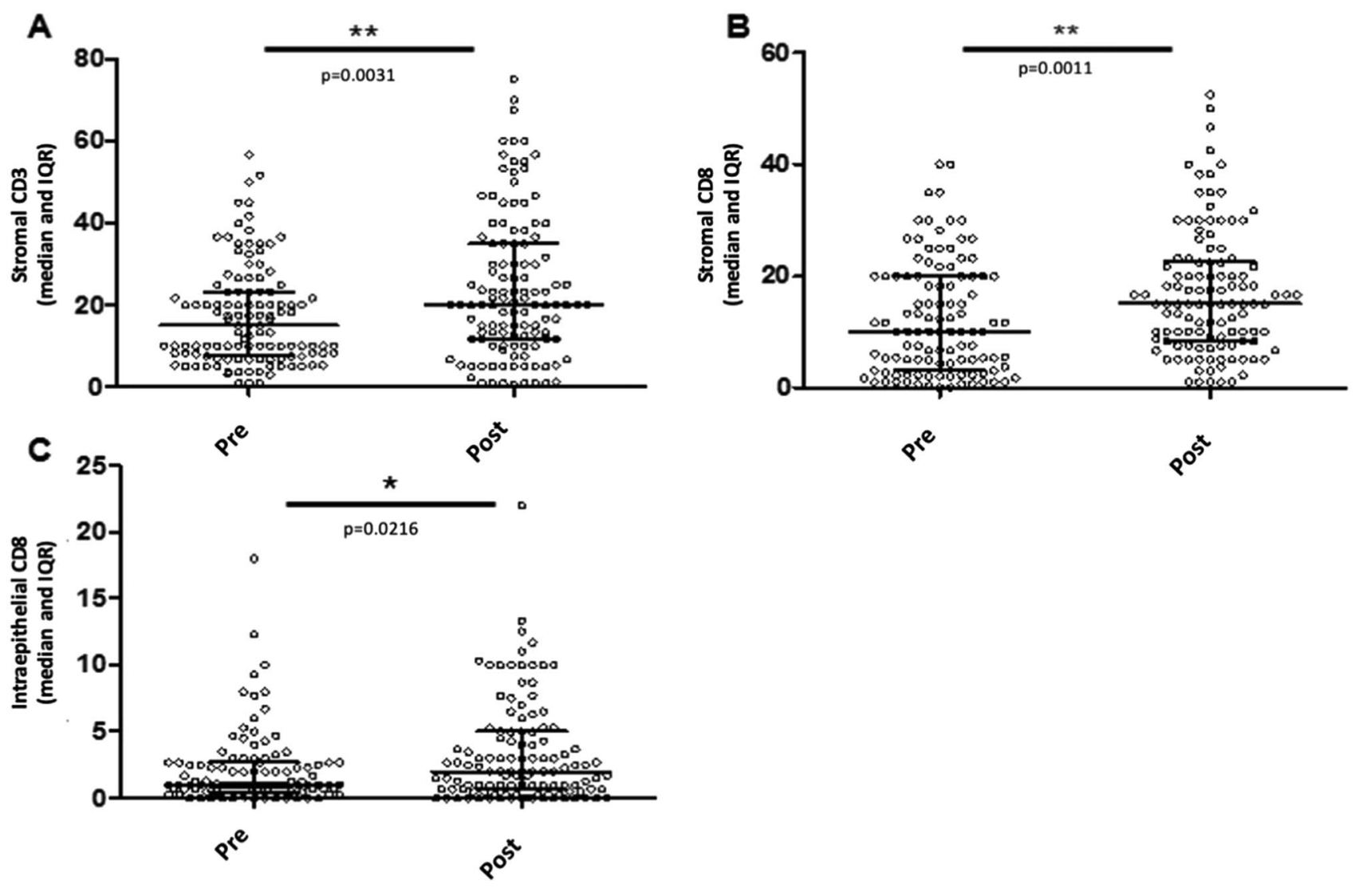

Fig. 1 Comparison of immune cell infiltrates in specimens of EOC patients prior and after neoadjuvant chemotherapy. a Changes in stromal CD3+ TILs (\%); b, c changes in stromal (\%) and intra-epithelial (/HPF) CD8+ TILs

showed diminishing levels, including $15 \%$ becoming completely ieFOXP3-negative post NACT (Fig. 4a-f).

Given the heterogeneous patterns of change in TIL subpopulations and the fact that the balance of immune-reactive versus immune-tolerant cells may be more relevant to deciphering the tumor immune contexture, the ratios of $\mathrm{CD} 8+1$ FOXP3+, CD4+/FOXP3+, and CD3+/FOXP3+ in individual tumors were derived. Overall, the median of $\mathrm{CD} 8+/$ FOXP3+ ratios increased significantly post NACT in the stromal $(p=<0.0001)$ and intra-epithelial compartments $(p=0.0007)$ as well as the median of intra-epithelial CD3+l FOXP3+ ratios $(p=0.006)$ favoring anti-tumor immunity. Also, the proportion of tumors demonstrating a favorable ratio (e.g., ratio of effector to suppressor TILs $>1$ ) increased post NACT from $71 \%$ to $95 \%$ (Chi square $<0.0001$ ) and from $48 \%$ to $78 \%$ (Chi square $<0.0001$ ) for stromal and ieCD8+/FOXP3, respectively (Table 1 ). With regards to CD4+/FOXP3+ ratios, the proportion of tumors showing an intra-epithelial CD4+/FOXP3+ ratio $>1$ increased from $42 \%$ to $60 \%$ (Chi square $=0.002$ ) with NACT, again suggesting that NACT shifted the balance towards effector versus suppressor T-cells. Stromal CD4+/FOXP3+ ratio did not change significantly post NACT (Table 1).

\section{Changes in CD68+ and CD163+ macrophages with NACT}

Subsets of TAMs were evaluated using CD68, a non-specific macrophage marker, and CD163, a putative marker for M2-polarized pro-tumoral macrophages. At diagnosis, tumors showed a greater prevalence of CD163+ than CD68+ TAMs. The median CD68+ score was $2.5(N=71$, range 0-30\%) compared to a median CD163+ score of 10 ( $N=74$, range $0-45 \%)$ suggesting that in treatment naïve EOC the macrophage compartment is primarily driven by pro-tumoral M2-like cells.

Post NACT, infiltration by CD68+ TAMs significantly increased to a median of $10(N=73, p=0.0003$, Supplementary Fig. 2a), while no significant overall change in median CD163+ TAMs was noted (median: $10, N=72$, $p=\mathrm{NS}$, Supplementary Fig. 2b). But as for FOXP3+, when considering paired samples from individual patients, the impact of NACT on CD163+ infiltration was variable with $42 \%$ showing an increase in CD163+ cells post NACT, 
A

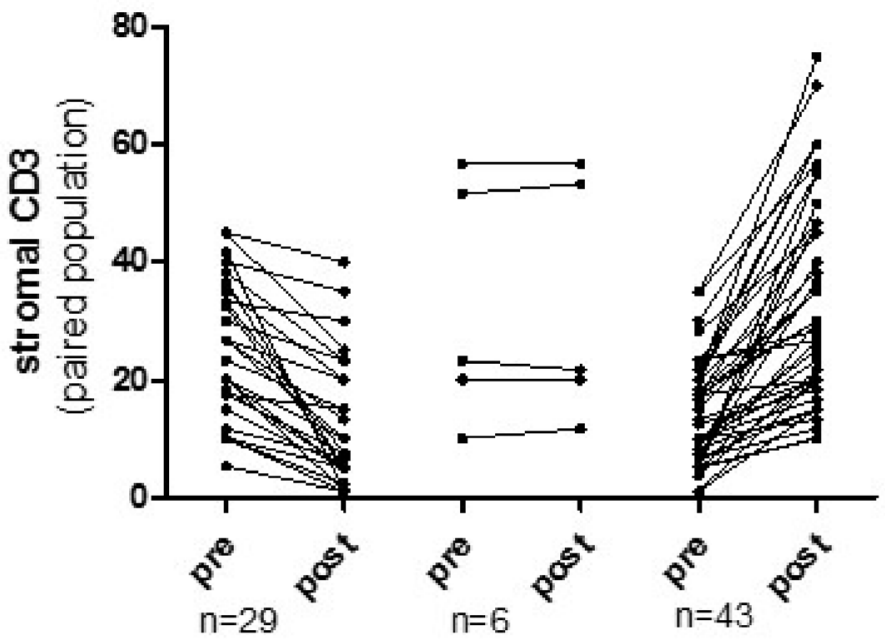

B

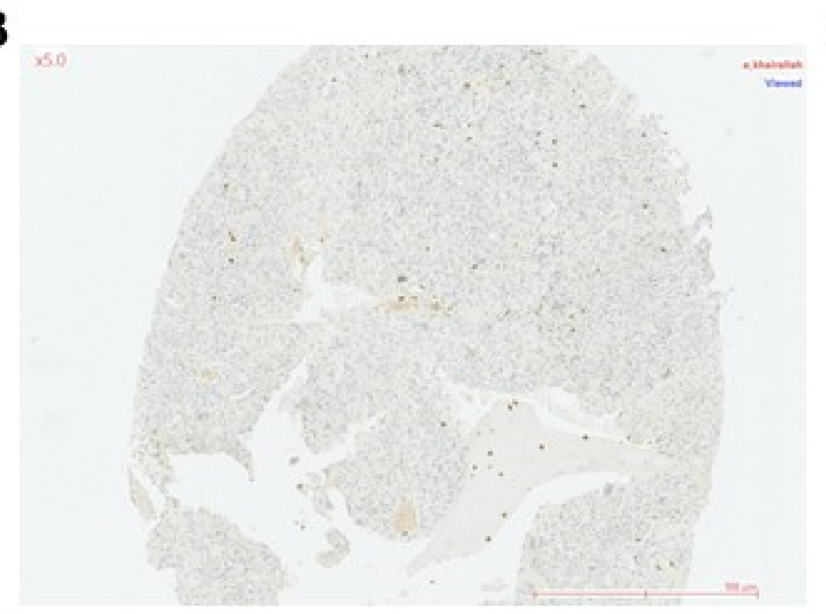

D

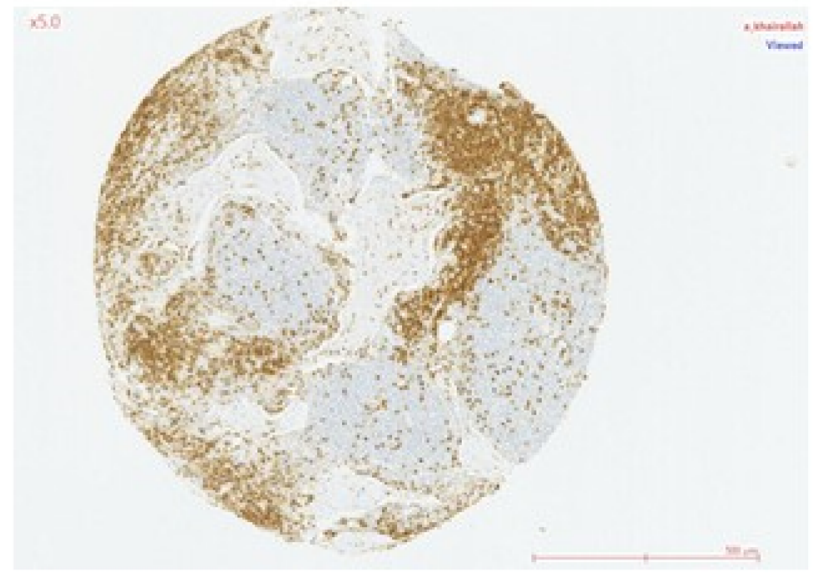

Fig. 2 a changes in CD3 expression in immune cells in paired preand post-NACT tumors, in the stromal compartment (\%). CD3 expression in paired tumors from a single patient showing an increase
C

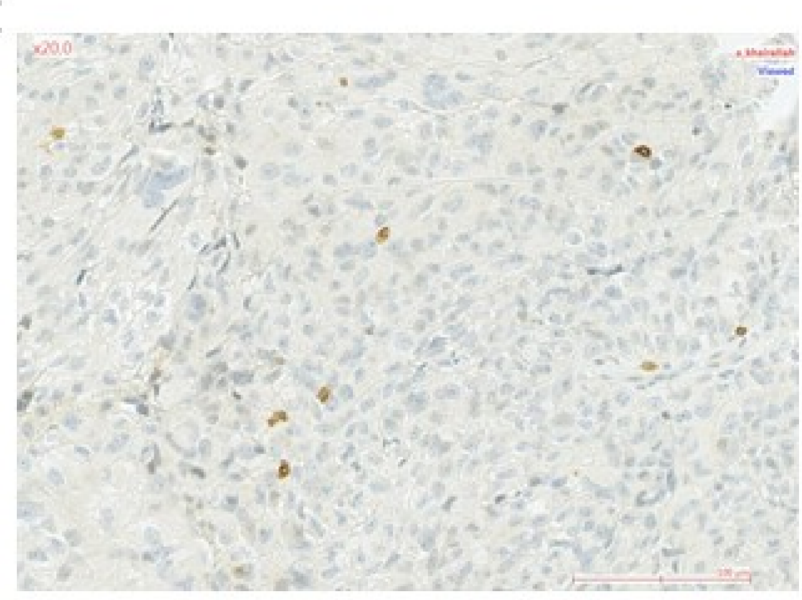

$E$

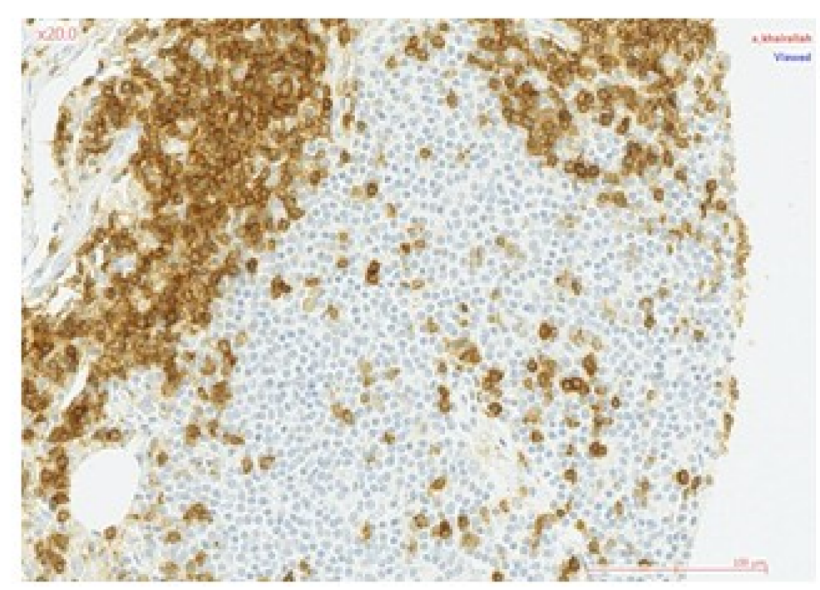

in $\mathrm{CD} 3$ expression after therapy. Pre-NACT, $\mathrm{B}(\times 5), \mathrm{C}(\times 20)$ and postNACT $D(\times 5), E(\times 20)$ 


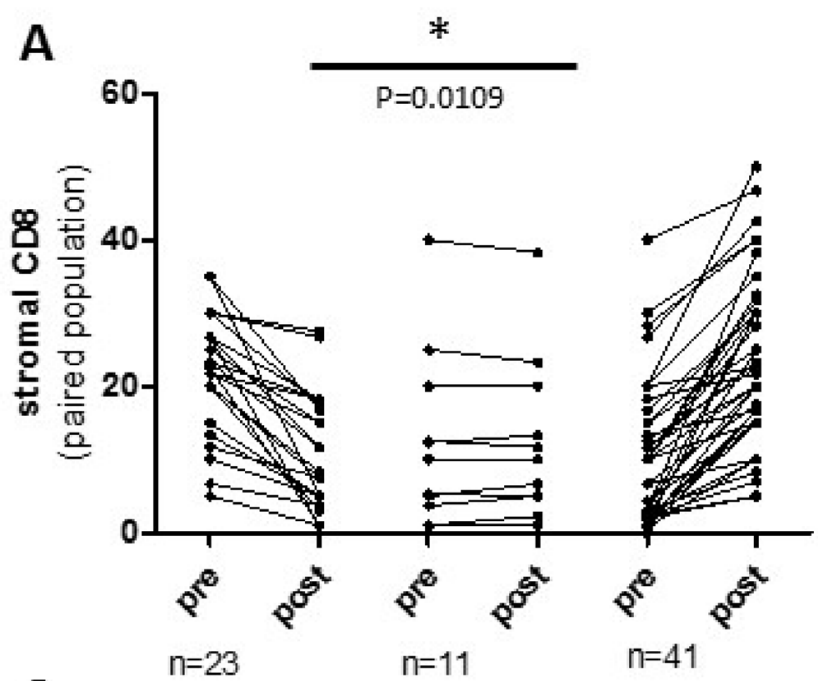

C

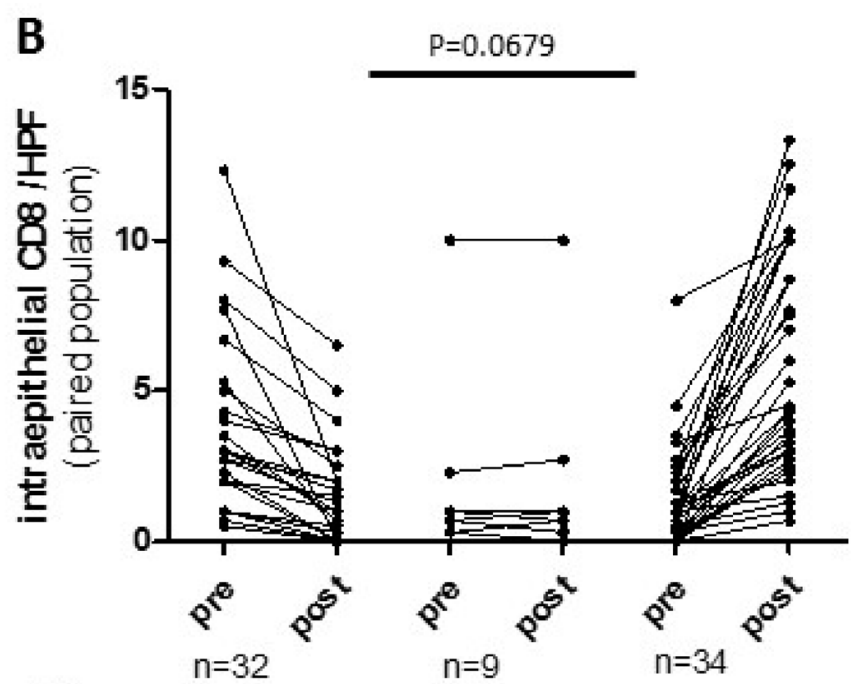

D

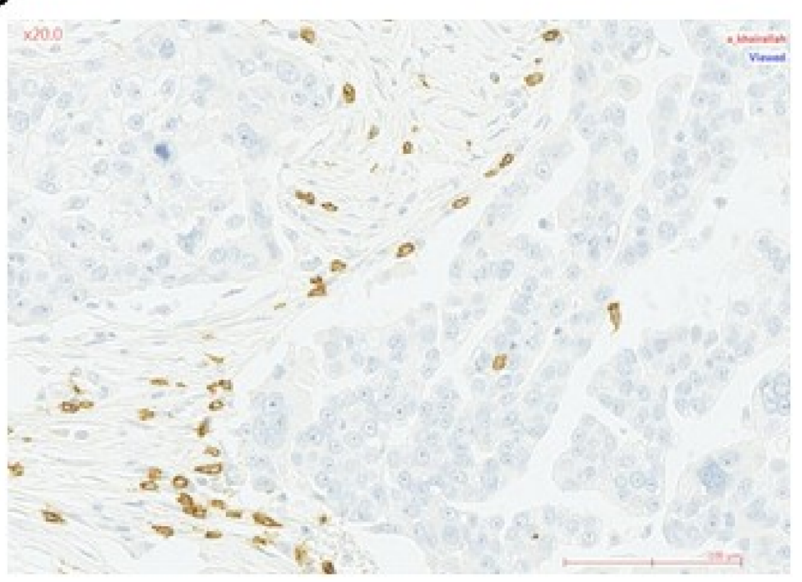

E

$\mathbf{F}$

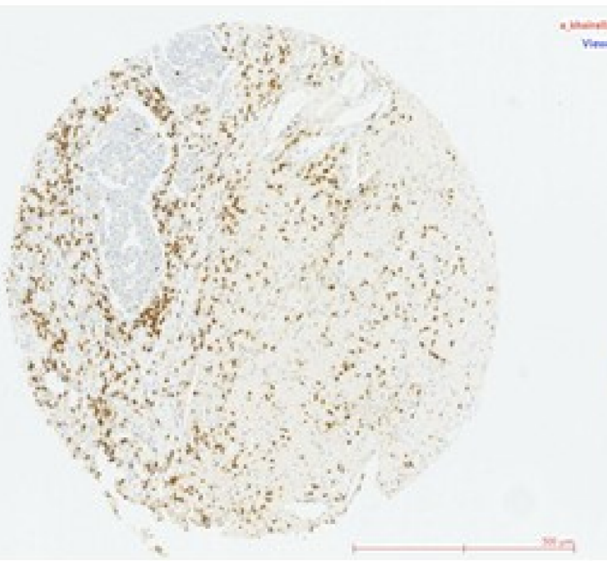

Fig. 3 Changes in CD8 expression in immune cells in paired preand post-NACT tumors; $\mathbf{a}$ in the stromal compartment (\%) and $\mathbf{b}$ in the intra-epithelial compartment (/HPF). CD8 expression in paired

tumors from a single patient showing an increase of CD8 expression after therapy. Pre-NACT, $\mathrm{C}(\times 5), \mathrm{D}(\times 20)$ and post-NACT $\mathrm{E}(\times 5)$, $\mathrm{F}(\times 20)$ 

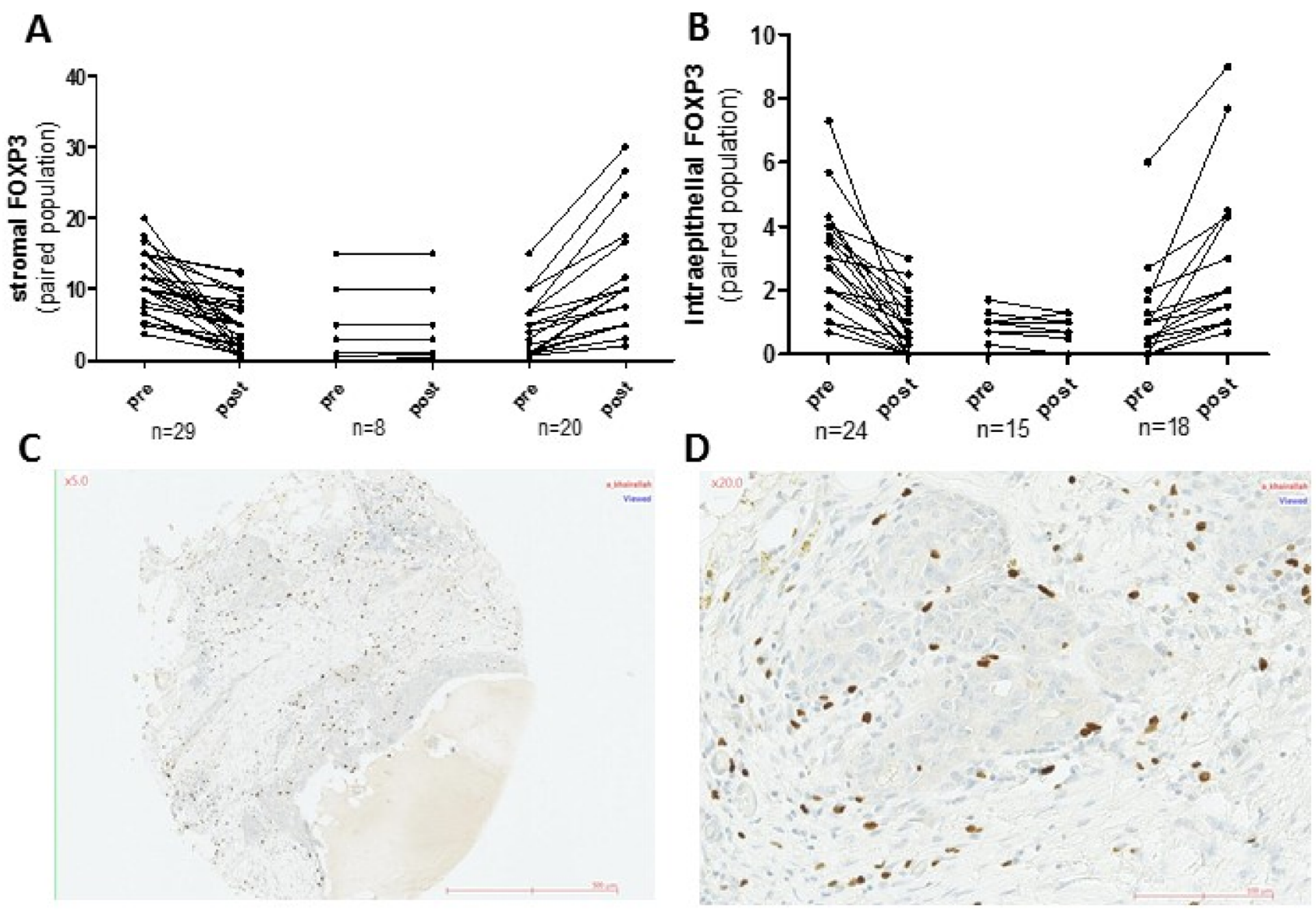

$E$

$\mathbf{F}$

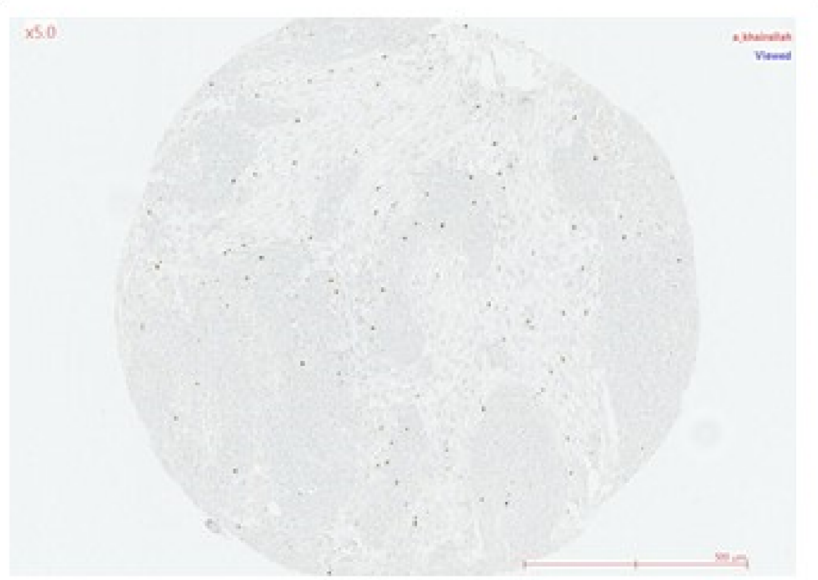

Fig. 4 Changes in FOXP3 expression in immune cells in paired preand post-NACT tumors; $\mathbf{a}$ in the stromal compartment (\%) and $\mathbf{b}$ in the intra-epithelial compartment (/HPF). FOXP3 expression in paired

tumors from a single patient showing a decrease in FOXP3 expression after therapy. Pre-NACT, $\mathrm{C}(\times 5), \mathrm{D}(\times 20)$ and post-NACT $\mathrm{E}(\times 5)$, $\mathrm{F}(\times 20)$

$46 \%$ showing a decrease, and $12 \%$ showing stable levels (Supplementary Fig. 2d). As a result, when considering the ratio of CD68+/CD163+ TAMs in individual tumors, there was a significant change in the balance of CD68+ to CD163+ macrophages with NACT. At diagnosis, the

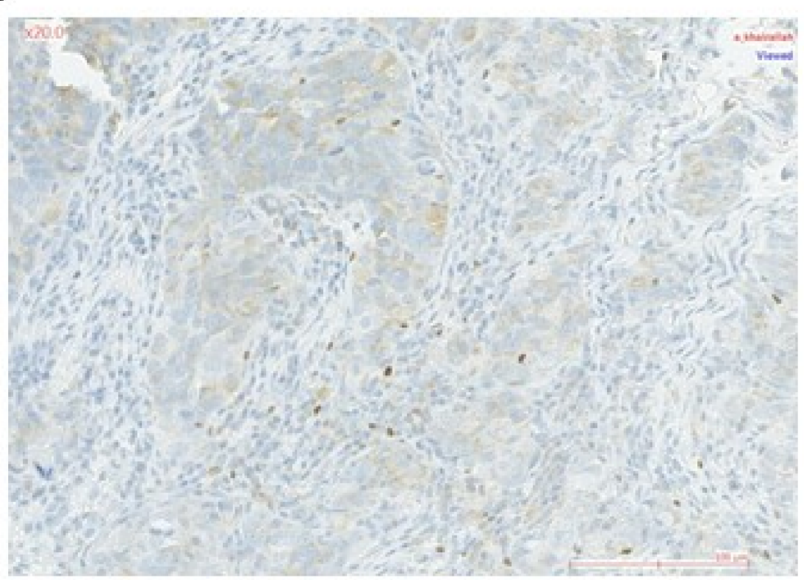

macrophage infiltration was dominated by CD163+ cells;

however, after NACT the proportion of tumors showing a greater infiltration of CD68 than CD163 macrophages increased significantly by over twofold from $14 \%$ to $35 \%$ (Chisquare- $p=0.0003$, Table 1 ). 
Table 1 Proportion of tumors showing a favorable effector to suppressor ratio (e.g., ratio > 1) pre-NACT and post-NACT

\begin{tabular}{|c|c|c|c|c|c|}
\hline \multirow[t]{2}{*}{ Ratios } & \multicolumn{2}{|c|}{ Pre-NACT } & \multicolumn{2}{|c|}{ Post-NACT } & \multirow[t]{2}{*}{$P$ value (chi square) } \\
\hline & $N$ & $\begin{array}{l}\% \text { tumors } \\
\text { with } \\
\text { ratio }>1\end{array}$ & $N$ & $\begin{array}{l}\% \text { tumors } \\
\text { with } \\
\text { ratio }>1\end{array}$ & \\
\hline
\end{tabular}

\begin{tabular}{|c|c|c|c|c|c|}
\hline \multicolumn{6}{|c|}{ Stromal CD8/FOXP3 } \\
\hline$>1$ & 60 & $71 \%$ & 95 & $95 \%$ & $<0.0001$ \\
\hline$\leq 1$ & 24 & & 5 & & \\
\hline \multicolumn{6}{|c|}{ ieCD8/FOXP3 } \\
\hline$>1$ & 34 & $48 \%$ & 60 & $78 \%$ & $<0.0001$ \\
\hline$\leq 1$ & 37 & & 17 & & \\
\hline \multicolumn{6}{|c|}{ Stromal CD4/FOXP3 } \\
\hline$>1$ & 46 & $54 \%$ & 51 & $49 \%$ & NS \\
\hline$\leq 1$ & 39 & & 53 & & \\
\hline \multicolumn{6}{|c|}{ ieCD4/FOXP3 } \\
\hline$>1$ & 30 & $42 \%$ & 48 & $60 \%$ & 0.002 \\
\hline$\leq 1$ & 41 & & 32 & & \\
\hline \multicolumn{6}{|c|}{ CD68/CD163 } \\
\hline$>1$ & 8 & $14 \%$ & 24 & $35 \%$ & 0.0003 \\
\hline$\leq 1$ & 52 & & 45 & & \\
\hline
\end{tabular}

\section{Correlation of TIL and TAM subsets with clinical outcome}

When considering standard prognostic factors (age, stage, BRCA status and completeness of resection), only completeness of resection (CC0 versus other) was significantly associated with PFS (HR $=0.31 ; p<0.0001)$. Contrary to the literature showing that density of CD3+ and CD8+ TILs pre-treatment is strongly associated with survival $[5,14]$, in our study focusing on patients with unresectable OC treated with NACT, none of the T-cell or macrophage subsets, in either stromal or intra-epithelial compartment, correlated to PFS in pre-NACT samples. The prognostic impact of levels of immune infiltration post NACT was examined next. High stromal FOXP3 was the only immune marker associated with PFS (HR $=1.7 ; p=0.03)$, but not OS. Neither CD3+, CD8+, CD4+ TILs, and CD68+ TAM nor CD163+ TAM showed any significant correlation with PFS or OS after neoadjuvant chemotherapy.

\section{Integrating the balance of immune-reactive to immune-tolerant subpopulations for prognostic information}

Given recent reports that the balance between effector and suppressor immune subsets may offer more useful prognostic information [5, 23-25], we evaluated the correlation between ratios of $\mathrm{CD} 8+/ \mathrm{FOXP} 3+, \mathrm{CD} 3+/ \mathrm{FOXP} 3+, \mathrm{CD} 4+/$ FOXP3+, and CD68+/CD163+ and survival in tumor samples at diagnosis and after NACT. None of the ratios were prognostic in pre-treated samples. However, using the median value as a cutoff $(\geq 3)$, a high stromal CD8+l FOXP3+ ratio post NACT was associated with a significant improvement in PFS (HR $=0.57 ; p=0.009$, Fig. 5a). In fact, the more the balance in the stromal tumor microenvironment post NACT shifted in favor of CD8+ over FOXP3+ TILs, the better the survival (test for trend, $p=0.01$ ), such that with a cutoff of $\geq 10$, a high stromal CD8+/FOXP3 post NACT was associated with a significant improvement in both PFS (HR $=0.48$; median: 19 vs. $31 \mathrm{mo}, p=0.005$, Fig. $5 b)$ and $\mathrm{OS}(\mathrm{HR}=0.49$; median: 37 vs. $51 \mathrm{mo}, p=0.03$, Fig. 5c). Post NACT CD8+/FOXP3+ ieTIL cell ratio was not correlated with clinical outcome.

Similarly, PFS was significantly increased in tumors with a high stromal $\mathrm{CD} 3+/ \mathrm{FOXP} 3+$ ratio post NACT $(\mathrm{HR}=0.6, p=0.02)$. High stromal CD4+/FOXP3 also significantly predicted improved PFS $(\mathrm{HR}=0.64 ; p=0.04)$ and $\mathrm{OS}(\mathrm{HR}=0.46 ; p=0.003)$; again, the higher the ratio, the greater the impact on OS $(p=0.01)$. High CD68+/CD163+ ratio showed a trend for improved PFS and OS, but neither were significant.

On multivariate analysis, after adjusting for completeness of surgery, the ratio of stromal CD8+/FOXP3 post NACT remained significantly associated with $\mathrm{PFS}(\mathrm{HR}=0.62$; $p=0.03)$, while the ratio of stromal CD4+/FOXP3+ post NACT was significantly predictive of $\mathrm{OS}(\mathrm{HR}=0.46$; $p=0.003$ ).

\section{Discussion}

We have previously reported that NACT had a marked impact on the tumor immune microenvironment, resulting in significant increases in TIL infiltration and PDL1 expression [21]. In the current study, we sought to characterize which immune subpopulations were actually recruited to the tumor bed, including T-cells and macrophages with a particular focus on the balance of effector to suppressor cells.

We have demonstrated that NACT significantly altered the balance of immune effector to suppressor T-cells and macrophages in favor of anti-tumor immunity. Overall, NACT was associated with significant increases in antitumor CD8+, CD3+ TILs, and CD68+ macrophages and had a more variable impact on suppressive FOXP3+ TILs and CD163+ macrophages. When considering ratios of effector to suppressor immune subsets in individual patients, we observed a significant increase in the proportion of tumors showing favorable ratios of $\mathrm{CD} 8+/ \mathrm{FOXP} 3+$ stromal and ieTILs $(p<0.0001)$, CD4+/FOXP3+ ieTILs $(\mathrm{p}=0.002)$, and CD68+/CD163+ macrophages $(p=0.0003)$ post NACT. Importantly, high CD8+/FOXP3+, CD4+/FOXP3+, and $\mathrm{CD} 3+/ \mathrm{FOXP} 3+$ post NACT were significantly predictive 
of both improved PFS and OS, while high CD68+/CD163+ post NACT was non-significantly associated with improved PFS. In contrast, baseline ratios were not prognostic. Taken together, these data suggest that the balance of immunetolerant to reactive cells in the residual tumor post NACT may be a significant driver of increased survival and that the prognosis of women with advanced EOC may be further improved by harnessing anti-tumor immunity during or after primary treatment.

We were unable to analyze the correlation between these ratio and response rates without risking significant bias. Indeed, response to chemotherapy is notoriously difficult to objectively measure in EOC. Peritoneal disease is poorly visualized on CT scans, Ca125 diminishes in $90 \%$ of patients, and scores such as the sugarbaker score are limited to frequent incomplete evaluation of the abdomen during diagnostic laparoscopy resulting in truncated scores. The chemotherapy response score (CRS) is an interesting option, and we attempted to perform it retrospectively; unfortunately, the block selected for the TMA construction was the one containing the most viable tumor tissue, frequently an omental biopsy, and is also the block that is recommended for the CRS

Recent evidence suggests that antineoplastic agents may exert therapeutic effects by increasing the host's immune anti-tumor response via a host of mechanisms. Anthracyclines or oxaliplatin have been shown to induce immunogenic cell death (ICD), thereby activating anti-tumor immunity [26]. With regard to cytotoxics conventionally used in ovarian cancer, neoadjuvant cisplatin increased the intra-tumoral trafficking of CD4 and CD8 T-cells in both mice models and patients with esophageal cancer [27], while paclitaxel augmented cellular immunity in patients with advanced non-small cell lung cancer by increasing circulating CD8 T-cells and IL-2-secreting CD4 T-cells [28]. Taxanes have also been reported to specifically impair the viability of FOXP $3^{+}$Treg cells [29] or to polarize macrophages toward an M1-like phenotype and to selectively kill myeloid-derived suppressor cells while sparing the M1-like macrophages [30]. Taken together, these data support that neoadjuvant platinum- and taxane-based treatment may indeed modulate the tumor immune microenvironment.

We showed that NACT resulted in significant increases in total and cytotoxic T-cells. These results are in agreement with prior studies in EOC [8, 23, 31, 32]. Our study showed a significant increase in CD68+ TAMs after chemotherapy. In line with our findings, DeNardo et al reported that cytotoxics, such as cisplatin or paclitaxel, favored recruitment of macrophages to neoplastic lesions and potentiated their cytotoxicity [33, 34].

Despite significant increases in $\mathrm{CD} 3+, \mathrm{CD} 8+$, and CD68+ cells post NACT, no immune subsets on their own were prognostic. This is in line with previous studies also failing to demonstrate a significant correlation between increased CD8+ or CD68+ cells post NACT and survival $[8,23,31,32]$.

Studies have demonstrated that accumulation of FOXP3+ regulatory T-cells in EOC limits anti-tumor immunity and favors tumor cell growth, thereby reducing patient survival.

In the current study, there was no significant change in median FOXP3+ levels. However, medians in the whole group fail to accurately capture the heterogeneous immune responses to NACT. When looking at paired samples from individual patients, half showed decreasing FOXP3+ TILs and FOXP3+ infiltration post NACT in individual patients was significantly correlated with outcome: patients with low FOXP3+ TILs post NACT had significantly prolonged PFS $(\mathrm{HR}=0.520 ; p=0.005)$. The same inter-patient variability in immune response to NACT was observed across all TIL and macrophage subsets (Fig. 1) and likely reflects heterogeneity in host and tumor factors in patients with advanced EOC. These results underscore the critical importance of evaluating the post-NACT tumor in individual patients in order to integrate data on the diagnostic tumor immune profile and its evolution with chemotherapy [21].

The balance between anti-tumor versus pro-tumor immune cells in individual patients post NACT was most significantly correlated with outcome. Increasing data suggest that cytotoxics can have a concerted effect both recruiting and activating immune effectors while inhibiting immune-tolerant subpopulations [34].

In the current study, high CD8+/FOXP3+ T-cell ratio after NACT was associated with significantly better survival. Using the median as a cut-off, high CD8+/FOXP3+ post NACT predicts improved PFS (HR $=0.59 ; p=0.017)$. Importantly, the positive prognostic value of $\mathrm{CD} 8+1$ FOXP3+ increases as the ratio increases, so that using a cut-off of $>10$, high CD8+/FOXP3+ is associated with a significant 12 months improvement in PFS $(\mathrm{HR}=0.48$; 19 vs 31 months, $p=0.005$ ) and a significant increase in OS (HR $=0.49$; median: 37 vs. 51 months, $p=0.03$ ). After NACT, it is the combination of high CD8+ TILs which recognize foreign antigens on tumor cells and kill it by inducing the release of perforin and granzyme, associated with decreased FOXP3+ TILs, which have a critical role in suppressing anti-tumor immunity, leading to improved survival. And the greater the difference between these two subsets, the more the outcome is improved. Similar observations were made in breast cancer patients treated with NACT where a high CD8+/FOXP3+ ratio in residual tumors was prognostic [35]. Granzyme B is a granule-associated protein crucial for cytolytic function constitutively expressed by NK cells and activated CD8+ cells. Polcher et al. [23] showed a strong correlation between high granzyme $\mathrm{B}+/ \mathrm{FOXP} 3+$ ratio post NACT and improved PFS among 30 paired EOC samples. Together, these data support the prognostic relevance of the 

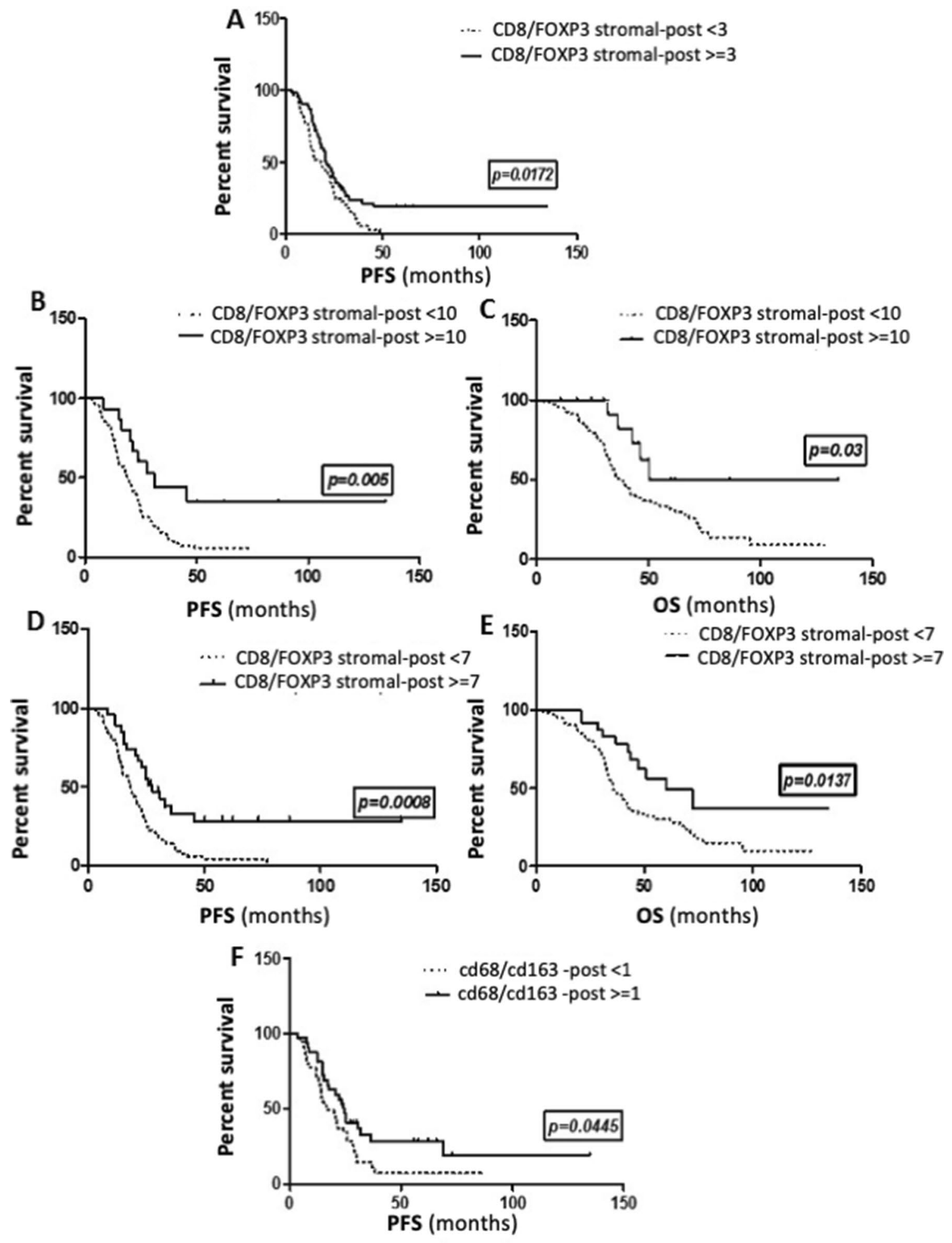

Fig. 5 Kaplan-Meier curves showing the correlation of the ratios of effector to tolerant immune infiltrates with survival in ovarian cancer. Post-NACT stromal CD8/FOXP3 ratio and PFS, with the median as a cut-off (a); with a cut-off of 10 (b); and OS with a cut-off of 10 (c).
Post-NACT stromal CD3/FOXP3 ratio and PFS (d) and OS (e) with a cut-off of 7. Post-NACT CD68/CD163 ratio and PFS with median as a cut-off (f) 
balance between immune activator and suppressor T-cells recruited to the tumor bed after NACT.

Similarly, the differential recruitment of subsets of macrophages to the tumor microenvironment provided the most prognostic information. Overall, CD68+ macrophages increased significantly post NACT, while median CD163+ remained stable; however, the pattern of change in CD163+ showed significant inter-patient variability. Macrophages can be classically (M1) or alternatively (M2) activated. M1 macrophages produce pro-inflammatory cytokines such as IL-12 and IL-23 or nitric oxide and demonstrate antigen-presenting activity, thus promoting tumor cell killing. Robust IHC markers for M1 markers are lacking; however, CD68 is a non-specific macrophage marker. In contrast, M2 macrophages which release immunosuppressive cytokines/chemokines are involved in immune tolerance and promote tumor growth and metastasis. The CD163 antigen is strictly expressed in monocytes/macrophages and is one of the markers used to identify M2 macrophages [36]. High levels of CD163+ macrophages in EOC samples at diagnosis have been shown to predict poor prognosis [37]. However, there is increasing data to suggest that it is in fact the balance between M1 and M2 subsets that is the most relevant. A high CD163+/CD68+ ratio at diagnosis has been shown to be a stronger predictor of OS, than CD163+ alone [37] and Zhang et al. demonstrated that a high M1/M2 ratio was associated with extended survival in ovarian cancer, whereas neither subtype alone was prognostic [38]. In the current study, CD163+ macrophages post NACT on their own did not predict outcome probably due to the tremendous overall increase in CD68+ cells, including M1 macrophages, which may counteract the immune-suppressive activity of CD163+ cells. Again, it was the balance of immunereactive versus tolerant macrophages post NACT that was most informative. Patients with tumors showing a high CD68+/CD163+ ratio post NACT had a slight improvement in PFS (16.5 vs 24 months, $p=0.044$ ), but lacked significance due to small numbers.

Despite recent meta-analyses providing evidence that high intra-epithelial CD3+, CD8+, or CD103+ TILs [39] and high CD68+/CD163+ ratios in EOC samples at diagnosis are predictive of survival [16], we could find no association between immune parameters at diagnosis and PFS or OS. Consistent with our findings, no association of preNACT CD3+, CD8+, FOXP3+, CD20+, or PDL1+ TILs with prognosis has been documented in several other studies $[23,31,32]$. Some aspects contributing to these conflicting results may include the method of assessing TILs, the use of different antibodies, as well as disease stage, immune status, and treatment pathways of the patients included. Indeed, ours and other studies showing no association of immune subsets at diagnosis with prognosis included only patients with bulky disease treated with NACT, while patients included in the meta-analyses were largely treated with upfront surgery. Advanced disease or neoadjuvant treatment may influence the prognostic value of baseline immune parameters, making those of post NACT more relevant.

In this report, stromal TILs offered the most prognostic information. Previous studies have largely described correlations between intra-epithelial TILs and survival in EOC. However, there is currently no standardized approach to evaluate TILs in EOC; the difference between intraepithelial/stromal/intra-tumoral/peritumoral TILs is poorly defined and often mingled. In contrast, in breast cancer, a recommended method of evaluation has been published by the international TILs Working Group where both stromal TILs and intra-epithelial TILs have been well defined and assessed as independent parameters, both representing true intra-tumoral TILs. Based on recent studies on breast cancer, stromal TILs have emerged as a stronger, more reproducible prognostic marker than intra-epithelial TILs [22, 40-42]. We had chosen to use their recommended definitions and method of evaluation in our study to differentiate intraepithelial from stromal TILs and to evaluate stromal TILs.

In summary, the current study is, to our knowledge, the biggest study describing the impact of carboplatin/taxanebased NACT on the lymphocyte and macrophage composition of advanced epithelial ovarian cancer. In fact, we add more power to previous studies by showing that neoadjuvant chemotherapy has the capacity to alter the influx and phenotype of immune infiltrates by significantly increasing overall CD3+, CD8+ cytotoxic cells, and CD68+ TAMs and decreasing FOXP3+ regulatory TILs or CD163+ macrophages in nearly half the patients. Importantly, relative infiltration by immune subtypes post NACT was prognostic. Using the median as a cut-off, high CD8+/FOXP3+, high CD3+/FOXP3+, and high CD68+/CD163+ in treated samples were all significantly predictive of improved PFS. In addition, the more the ratio shifted in favor of effector versus regulatory $\mathrm{T}$-cells, the greater the prognostic benefit such that patients with $\mathrm{CD} 8+/ \mathrm{FOXP} 3+$ or $\mathrm{CD} 3+/ \mathrm{FOXP} 3+$ ratios greater than 10 and 7 , respectively, had significant improvements in both PFS and OS. These data support that in a subset of patients, NACT may modulate the equilibrium of immune subsets in favor of immune rejection, thus contributing to tumor eradication and improved outcomes. Moreover, the impact of NACT on the tumor immune microenvironment showed significant inter-patient variability. This likely reflects heterogeneity in tumor and host factors in advanced EOC and could have important implications for personalized immunotherapy. We recommend further studies on bigger cohorts to better elucidate the immune changes occurring at the tumor bed after NACT. In the future, an individualized approach based on the type of immune subpopulations and expression of co-regulatory molecules identified in tumor 
at interval debulking after NACT could be proposed and may represent a promising approach to harness anti-tumor immunity, eradicate minimal residual disease, and result in meaningful improvements in outcome for patients with advanced ovarian cancer.

Author contributions AL, AK: design, writing, and supervision. CG, ALF, SM: technical analysis. FBD: writing. SG, AM, BC, JM, EB, PM, PP: supervision. AD: statistical analysis.

Funding Supported by a grant for DUERTECC/EURONCO (Diplome Universitaire Européen de Recherche Translationnelle Et Clinique en Cancérologie) and by a grant from the French National Institute du Cancer, PAIR GYNECO.

Availability of data and materials Datasets generated for the current study are not publicly available considering confidentiality reasons. Anonymized data may be available from the corresponding author on justified request.

\section{Compliance with ethical standards}

Conflict of interest The authors declare no potential conflicts of interest.

Ethics approval All patients provided written consent authorizing the use of residual tumor tissue obtained during their routine diagnosis and treatment. Research project was approved by the Gustave Roussy R\&D committee. Study was performed in accordance with the Declaration of Helsinki.

Open Access This article is licensed under a Creative Commons Attribution 4.0 International License, which permits use, sharing, adaptation, distribution and reproduction in any medium or format, as long as you give appropriate credit to the original author(s) and the source, provide a link to the Creative Commons licence, and indicate if changes were made. The images or other third party material in this article are included in the article's Creative Commons licence, unless indicated otherwise in a credit line to the material. If material is not included in the article's Creative Commons licence and your intended use is not permitted by statutory regulation or exceeds the permitted use, you will need to obtain permission directly from the copyright holder. To view a copy of this licence, visit http://creativecommons.org/licenses/by/4.0/.

\section{References}

1. Siegel RL, Miller KD, Jemal A (2015) Cancer statistics, 2015. CA Cancer J Clin 65:5-29. https://doi.org/10.3322/caac.21254

2. Ferlay J, Soerjomataram I, Dikshit R, Eser S, Mathers C, Rebelo $M$ et al (2015) Cancer incidence and mortality worldwide: sources, methods and major patterns in GLOBOCAN 2012. Int J Cancer 136:E359-E386. https://doi.org/10.1002/ijc.29210

3. Vergote I, Tropé CG, Amant F, Kristensen GB, Ehlen T, Johnson $\mathrm{N}$ et al (2010) Neoadjuvant chemotherapy or primary surgery in stage IIIC or IV ovarian cancer. N Engl J Med 363:943-953. https ://doi.org/10.1056/NEJMoa0908806

4. Kehoe S, Hook J, Nankivell M, Jayson GC, Kitchener H, Lopes $T$ et al (2015) Primary chemotherapy versus primary surgery for newly diagnosed advanced ovarian cancer (CHORUS): an open-label, randomised, controlled, non-inferiority trial. Lancet 386:249-257. https://doi.org/10.1016/S0140-6736(14)62223-6

5. Sato E, Olson SH, Ahn J, Bundy B, Nishikawa H, Qian F et al (2005) Intraepithelial CD8+ tumor-infiltrating lymphocytes and a high $\mathrm{CD} 8+$ /regulatory $\mathrm{T}$ cell ratio are associated with favorable prognosis in ovarian cancer. Proc Natl Acad Sci USA 102:1853818543. https://doi.org/10.1073/pnas.0509182102

6. Clarke B, Tinker AV, Lee C-H, Subramanian S, van de Rijn M, Turbin D et al (2009) Intraepithelial T cells and prognosis in ovarian carcinoma: novel associations with stage, tumor type, and BRCA1 loss. Mod Pathol 22:393-402. https://doi.org/10.1038/ modpathol.2008.191

7. Hamanishi J, Mandai M, Iwasaki M, Okazaki T, Tanaka Y, Yamaguchi $\mathrm{K}$ et al (2007) Programmed cell death 1 ligand 1 and tumorinfiltrating CD8+ T lymphocytes are prognostic factors of human ovarian cancer. Proc Natl Acad Sci USA 104:3360-3365. https:// doi.org/10.1073/pnas.0611533104

8. Wouters MCA, Komdeur FL, Workel HH, Klip HG, Plat A, Kooi NM et al (2016) Treatment regimen, surgical outcome, and T-cell differentiation influence prognostic benefit of tumor-infiltrating lymphocytes in high-grade serous ovarian cancer. Clin Cancer Res 22:714-724. https://doi.org/10.1158/1078-0432.CCR-15-1617

9. Matsushita N, Ghazizadeh M, Konishi H, Araki T (2003) Association of ovarian tumor epithelium coexpressing HLA-DR and CA-125 antigens with tumor infiltrating cytotoxic T lymphocytes. J Nippon Med Sch 70:40-44. https://doi.org/10.1272/jnms.70.40

10. Hamanishi J, Mandai M, Abiko K, Matsumura N, Baba T, Yoshioka Y et al (2011) The comprehensive assessment of local immune status of ovarian cancer by the clustering of multiple immune factors. Clin Immunol 141:338-347. https://doi. org/10.1016/j.clim.2011.08.013

11. Vermeij R, de Bock GH, Leffers N, Ten Hoor KA, Schulze U, Hollema $\mathrm{H}$ et al (2011) Tumor-infiltrating cytotoxic $\mathrm{T}$ lymphocytes as independent prognostic factor in epithelial ovarian cancer with wilms tumor protein 1 overexpression. J Immunother 34:516-523. https://doi.org/10.1097/CJI.0b013e31821e012f

12. Nielsen JS, Sahota RA, Milne K, Kost SE, Nesslinger NJ, Watson $\mathrm{PH}$ et al (2012) CD20+ tumor-infiltrating lymphocytes have an atypical CD27- memory phenotype and together with CD8+ $\mathrm{T}$ cells promote favorable prognosis in ovarian cancer. Clin Cancer Res 18:3281-3292. https://doi.org/10.1158/1078-0432. CCR-12-0234

13. Barnett JC, Bean SM, Whitaker RS, Kondoh E, Baba T, Fujii $S$ et al (2010) Ovarian cancer tumor infiltrating T-regulatory (T(reg)) cells are associated with a metastatic phenotype. Gynecol Oncol 116:556-562. https://doi.org/10.1016/j.ygyno.2009.11.020

14. Milne K, Köbel M, Kalloger SE, Barnes RO, Gao D, Gilks CB et al (2009) Systematic analysis of immune infiltrates in highgrade serous ovarian cancer reveals CD20, FoxP3 and TIA-1 as positive prognostic factors. PLoS ONE 4:e6412. https://doi. org/10.1371/journal.pone.0006412

15. Curiel TJ, Coukos G, Zou L, Alvarez X, Cheng P, Mottram P et al (2004) Specific recruitment of regulatory T cells in ovarian carcinoma fosters immune privilege and predicts reduced survival. Nat Med 10:942-949. https://doi.org/10.1038/nm1093

16. Yuan X, Zhang J, Li D, Mao Y, Mo F, Du W et al (2017) Prognostic significance of tumor-associated macrophages in ovarian cancer: a meta-analysis. Gynecol Oncol 147:181-187. https://doi. org/10.1016/j.ygyno.2017.07.007

17. Knutson KL, Maurer MJ, Preston CC, Moysich KB, Goergen K, Hawthorne KM et al (2015) Regulatory T cells, inherited variation, and clinical outcome in epithelial ovarian cancer. Cancer Immunol Immunother 64:1495-1504. https://doi.org/10.1007/ s00262-015-1753-x

18. Galluzzi L, Buqué A, Kepp O, Zitvogel L, Kroemer G (2015) Immunological effects of conventional chemotherapy and 
targeted anticancer agents. Cancer Cell 28:690-714. https://doi. org/10.1016/j.ccell.2015.10.012

19. Lake RA, Robinson BWS (2005) Immunotherapy and chemotherapy-a practical partnership. Nat Rev Cancer 5:397-405. https:// doi.org/10.1038/nrc1613

20. Ghiringhelli F, Larmonier N, Schmitt E, Parcellier A, Cathelin D, Garrido $\mathrm{C}$ et al (2004) CD4+ CD25+ regulatory T cells suppress tumor immunity but are sensitive to cyclophosphamide which allows immunotherapy of established tumors to be curative. Eur J Immunol 34:336-344. https://doi.org/10.1002/eji.200324181

21. Mesnage SJL, Auguste A, Genestie C, Dunant A, Pain E, Drusch $F$ et al (2017) Neoadjuvant chemotherapy (NACT) increases immune infiltration and programmed death-ligand 1 (PD-L1) expression in epithelial ovarian cancer (EOC). Ann Oncol 28:651-657. https://doi.org/10.1093/annonc/mdw625

22. Salgado R, Denkert C, Demaria S, Sirtaine N, Klauschen F, Pruneri $G$ et al (2015) The evaluation of tumor-infiltrating lymphocytes (TILs) in breast cancer: recommendations by an International TILs Working Group 2014. Ann Oncol 26:259-271. https ://doi.org/10.1093/annonc/mdu450

23. Pölcher M, Braun M, Friedrichs N, Rudlowski C, Bercht E, Fimmers R et al (2010) Foxp3(+) cell infiltration and granzyme B(+)/ Foxp3(+) cell ratio are associated with outcome in neoadjuvant chemotherapy-treated ovarian carcinoma. Cancer Immunol Immunother 59:909-919. https://doi.org/10.1007/s00262-010-0817-1

24. Gao Q, Qiu S-J, Fan J, Zhou J, Wang X-Y, Xiao Y-S et al (2007) Intratumoral balance of regulatory and cytotoxic $\mathrm{T}$ cells is associated with prognosis of hepatocellular carcinoma after resection. J Clin Oncol 25:2586-2593. https://doi.org/10.1200/ JCO.2006.09.4565

25. Galon J, Costes A, Sanchez-Cabo F, Kirilovsky A, Mlecnik $B$, Lagorce-Pagès $C$ et al (2006) Type, density, and location of immune cells within human colorectal tumors predict clinical outcome. Science 313:1960-1964. https://doi.org/10.1126/scien ce. 1129139

26. Zitvogel L, Kepp O, Kroemer G (2011) Immune parameters affecting the efficacy of chemotherapeutic regimens. Nat Rev Clin Oncol 8:151-160. https://doi.org/10.1038/nrclinonc.2010.223

27. Predina JD, Judy B, Aliperti LA, Fridlender ZG, Blouin A, Kapoor V et al (2011) Neoadjuvant in situ gene-mediated cytotoxic immunotherapy improves postoperative outcomes in novel syngeneic esophageal carcinoma models. Cancer Gene Ther 18:871-883. https://doi.org/10.1038/cgt.2011.56

28. Zhang L, Dermawan K, Jin M, Liu R, Zheng H, Xu L et al (2008) Differential impairment of regulatory $\mathrm{T}$ cells rather than effector T cells by paclitaxel-based chemotherapy. Clin Immunol 129:219229. https://doi.org/10.1016/j.clim.2008.07.013

29. Zhu Y, Liu N, Xiong SD, Zheng YJ, Chu YW (2011) CD4+ Foxp3+ regulatory T-cell impairment by paclitaxel is independent of toll-like receptor 4. Scand J Immunol 73:301-308. https:// doi.org/10.1111/j.1365-3083.2011.02514.x

30. Kodumudi KN, Woan K, Gilvary DL, Sahakian E, Wei S, Djeu JY (2010) A novel chemoimmunomodulating property of docetaxel: suppression of myeloid-derived suppressor cells in tumor bearers. Clin Cancer Res 16:4583-4594. https://doi.org/10.1158/10780432.CCR-10-0733

31. Böhm S, Montfort A, Pearce OMT, Topping J, Chakravarty P, Everitt GLA et al (2016) Neoadjuvant chemotherapy modulates the immune microenvironment in metastases of tubo-ovarian high-grade serous carcinoma. Clin Cancer Res 22:3025-3036. https://doi.org/10.1158/1078-0432.CCR-15-2657

32. Lo CS, Sanii S, Kroeger DR, Milne K, Talhouk A, Chiu DS et al (2017) Neoadjuvant chemotherapy of ovarian cancer results in three patterns of tumor-infiltrating lymphocyte response with distinct implications for immunotherapy. Clin Cancer Res 23:925934. https://doi.org/10.1158/1078-0432.CCR-16-1433

33. DeNardo DG, Brennan DJ, Rexhepaj E, Ruffell B, Shiao SL, Madden SF et al (2011) Leukocyte complexity predicts breast cancer survival and functionally regulates response to chemotherapy. Cancer Discov 1:54-67. https://doi.org/10.1158/2159-8274. CD-10-0028

34. Bracci L, Schiavoni G, Sistigu A, Belardelli F (2014) Immunebased mechanisms of cytotoxic chemotherapy: implications for the design of novel and rationale-based combined treatments against cancer. Cell Death Differ 21:15-25. https://doi.org/10.1038/ cdd.2013.67

35. Miyashita M, Sasano H, Tamaki K, Hirakawa H, Takahashi Y, Nakagawa $S$ et al (2015) Prognostic significance of tumor-infiltrating CD8+ and FOXP3+ lymphocytes in residual tumors and alterations in these parameters after neoadjuvant chemotherapy in triple-negative breast cancer: a retrospective multicenter study. Breast Cancer Res 17:124. https://doi.org/10.1186/s1305 8-015-0632-x

36. Kurahara $\mathrm{H}$, Shinchi $\mathrm{H}$, Mataki $\mathrm{Y}$, Maemura K, Noma H, Kubo F et al (2011) Significance of M2-polarized tumor-associated macrophage in pancreatic cancer. J Surg Res 167:e211-e219. https:// doi.org/10.1016/j.jss.2009.05.026

37. Lan C, Huang X, Lin S, Huang H, Cai Q, Wan T et al (2013) Expression of M2-polarized macrophages is associated with poor prognosis for advanced epithelial ovarian cancer. Technol Cancer Res Treat 12:259-267. https://doi.org/10.7785/tcrt.2012.500312

38. Zhang M, He Y, Sun X, Li Q, Wang W, Zhao A et al (2014) A high M1/M2 ratio of tumor-associated macrophages is associated with extended survival in ovarian cancer patients. J Ovarian Res 7:19. https://doi.org/10.1186/1757-2215-7-19

39. Li J, Wang J, Chen R, Bai Y, Lu X (2017) The prognostic value of tumor-infiltrating T lymphocytes in ovarian cancer. Oncotarget 8:15621-15631. https://doi.org/10.18632/oncotarget.14919

40. Hwang C, Lee S, Lee J, Kim K, Suh D, Kwon B et al (2019) Stromal tumor-infiltrating lymphocytes evaluated on H\&E-stained slides are an independent prognostic factor in epithelial ovarian cancer and ovarian serous carcinoma. Oncol Lett. https://doi. org/10.3892/ol.2019.10095

41. James FR, Jiminez-Linan M, Alsop J, Mack M, Song H, Brenton JD et al (2017) Association between tumour infiltrating lymphocytes, histotype and clinical outcome in epithelial ovarian cancer. BMC Cancer 17:657. https://doi.org/10.1186/s12885-017-3585-x

42. Denkert C, von Minckwitz G, Darb-Esfahani S, Lederer B, Heppner BI, Weber KE et al (2018) Tumour-infiltrating lymphocytes and prognosis in different subtypes of breast cancer: a pooled analysis of 3771 patients treated with neoadjuvant therapy. Lancet Oncol 19:40-50. https://doi.org/10.1016/S1470-2045(17)30904-X

Publisher's Note Springer Nature remains neutral with regard to jurisdictional claims in published maps and institutional affiliations. 